\title{
Diversity of Macrolichens in Bolampatti II Forest Range (Siruvani Hills), Western Ghats, Tamil Nadu, India
}

\author{
P. Balaji ${ }^{1}$ and G. N. Hariharan ${ }^{2}$ \\ ${ }^{1}$ Department of Botany, Dr. Ambedkar Government Arts College, Vyasarpadi, Chennai, Tamil Nadu 600 039, India \\ ${ }^{2}$ Lichen Ecology and Bioprospecting Laboratory, M. S. Swaminathan Research Foundation III Cross Street, \\ Taramani Institutional Area, Taramani, Chennai, Tamil Nadu 600 113, India
}

Correspondence should be addressed to P. Balaji; lichenbalaji@gmail.com

Received 26 June 2013; Accepted 4 August 2013

Academic Editors: A. R. Atangana and R. Rico-Martinez

Copyright (C) 2013 P. Balaji and G. N. Hariharan. This is an open access article distributed under the Creative Commons Attribution License, which permits unrestricted use, distribution, and reproduction in any medium, provided the original work is properly cited.

An annotated checklist of 103 macrolichen species is provided based on identification of specimens collected from three different vegetation types within the Bolampatti II forest range, Western Ghats, India. Among them, the dominant order is Lecanorales with 47 species, while the dominant family is Parmeliaceae with 40 species. The foremost genus is Usnea with 15 species.

\section{Introduction}

Nearly 80,000 species of fungi are available in nature [1]. Of these, approximately $17 \%$ are lichenized, forming symbioses with green algae (Chlorophyta, Viridiplantae) or the so called blue-green algae (Cyanobacteria, Bacteria). These relationships produce symbiotic organisms commonly called lichens [2]. Lichens are an outstandingly successful group of symbiotic organisms exploiting a wide range of habitats throughout the world. About 20,000 species of lichens are so far recorded across the globe, among which the Indian subcontinent harbours 2450 (12.25\%) species [3]. Tropical forests, because of their complexity and variety of microhabitats, usually harbour a rich diversity of lichens. Even though they are often small and inconspicuous, especially in the lowland forest, they may play a significant role in the forest ecosystem [4]. It has been estimated that $50 \%$ of the Indian lichen biota are currently undescribed [5]. In India, the comprehensive regional treatments (both ecological and systematic) on lichens are still not available, and a few cover areas like portions of Western Ghats (Nilgiri and Palni Hills, Himalayas, and North Eastern regions) [6, 7]. Still, many of the pristine Western Ghats ecosystems remain unexplored to list out the extent and type of distribution of lichens [8]. In view to explore such important organisms, the present study has studied the macrolichens from Bolampatti II forest range, Western Ghats. The primary objective of this paper is to enumerate the macrolichens and their distribution within the forest types of the Bolampatti II forest range.

\section{Materials and Methods}

2.1. Study Area. Bolampatti II forest range, Coimbatore district, Tamil Nadu (part of Western Ghats- $76^{\circ} 33^{\prime \prime}$ to $76^{\circ} 46^{\prime \prime} \mathrm{E}$ and $11^{\circ} 2^{\prime \prime}$ to $10^{\circ} 54^{\prime \prime} \mathrm{N}$ ), is a part of the Nilgiri Biosphere Reserve, is commonly known as Siruvani Hills (Figure 1), and is one of the biodiversity hotspots of the world. The study site is located west to Coimbatore city and north of the Walayar valley, is shaped like a horse-shoe opening eastwards, and covers an area of $197.66 \mathrm{~km}^{2}$. The Bolampatti valley consists of vegetation types progressively from lower to higher altitudes (east to west) dry deciduous forest (DDF) (4a/C2), moist deciduous forest (MDF), and western tropical evergreen forest (WTEF) $(1 \mathrm{a} / \mathrm{C} 3)[9,10]$. This valley drains eastwards into the Noyilar and its tributaries. The elevation ranges from $458 \mathrm{~m}$ (Noyilar base) to $1,986 \mathrm{~m}$ on Periyakunjiramalai at the southwest corner and $1,800 \mathrm{~m}$ on Vellingiri peak at the northwest. This hill receives a rainfall of 75-200 cm from the northeast monsoon during September to November, with a dry period of six months. The temperature ranges between $10^{\circ} \mathrm{C}$ during December and January and $40^{\circ} \mathrm{C}$ 


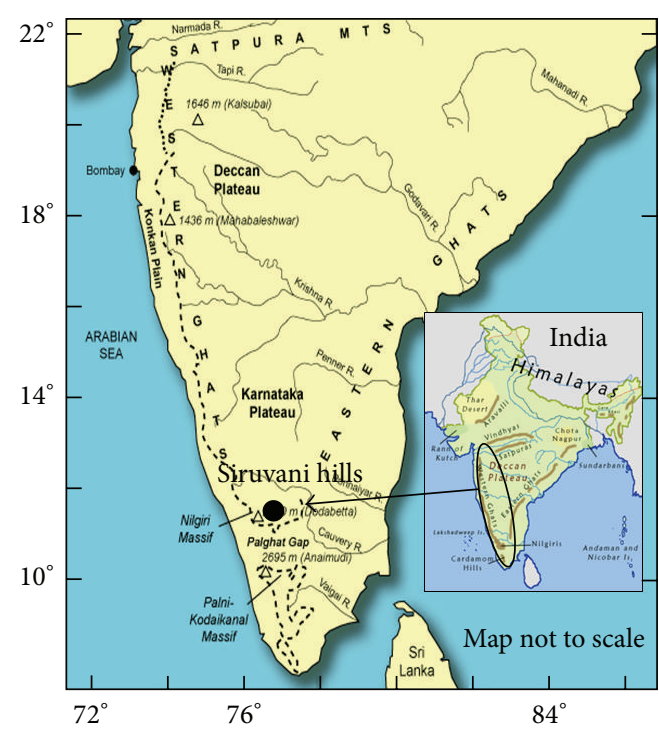

Figure 1: Map of Bolampatti II forest range (Siruvani Hills), Western Ghats.

during March to May. The older rock is of Precambrian origin with the formation of mainly Charnockite or Nilgiri gneiss. Soil is mainly of red type in the hills. The foothills generally have sandy loam. The Vellingiri peak is barren and rocky.

2.2. Research Methods. The lichen samples (approximately. 3000 specimens) were collected from various vegetation types, namely, dry deciduous forest (DDF), moist deciduous forest (MDF), and western tropical evergreen forest (WTEF) during 2001-2005 by the authors based on ocular survey on all possible lichen occurring substrates, such as tree bark, rocks, leaves, and soil. Lichens on fallen twigs were collected, since they represent the species that occur on canopy. Each sample was given a field number. The specimens were dried and incorporated into a reference collection that is preserved in the Lichen Ecology and Bioprospecting Laboratory, M. S. Swaminathan Research Foundation (MSSRF), Chennai. The specimens were identified based on the internal and external morphological, reproductive, and chemical features following recent literature [11-18], and lichen taxa are classified based on the systematic arrangement provided by Hawksworth et al. [19]. For each of the lichen species collected, their distribution with respect to growth form, substratum preference, and forest types were provided.

\section{Results and Discussion}

A total number of 103 species of macrolichens under 27 genera in 9 families in 3 fungal orders were recorded within the Siruvani hills (Table 1; Figures 2(a)-2(f)). Fungal order Lecanorales dominate with 47 species followed by Teloschistales (35) and Peltigerales (21). Out of 9 families of lichens, Parmeliaceae (40 species) is the largest family followed by Physciaceae (20), Collemataceae (16), Caliciaceae
(14), Ramalinaceae (6), and Lobariaceae (4), and three families were with single species each. The largest genus was Usnea with 15 species followed by Heterodermia and Parmotrema with 13 species each, Leptogium and Pyxine with 11 and 10 species, respectively.

Fourteen (14) genera were with single species each. Among the forest types studied, MDF contain 55 lichens followed by WTEF with 51 and DDF with 48 species, in which, 21 lichen species were specific to DDF while 24 to MDF and 21 to WTEF. Thirty-seven (14) lichen species were common to all the forest types. Seven (7) species were shared between DDF and MDF, while 6 species between DDF and WTEF, and 24 species between MDF and WTEF.

Among the species recorded, $92.2 \%$ of lichens were colonized on bark (corticolous), followed by $6 \%$ on rock (saxicolous), $0.8 \%$ on soil (terricolous), and a single lichen species both on bark and rock substratum. Bark colonizing lichens were maximum in both MDF \& WTEF (47\% each) followed by DDF (43\%). Rock colonizing lichens were maximum in MDF (4) followed by DDF (3) and WTEF (1). Single soil colonizing lichen Cladonia sp. was found in MDF. Lichen Parmotrema praesorediosum was found colonizing both on bark as well as on rock in DDF type.

The photobiont distribution showed $80 \%$ of lichens with Trebouxia and $20 \%$ of lichens with Nostoc sp. Trebouxia containing lichens were maximum in WTEF and MDF with $34 \%$ each, followed by DDF (32\%). Nostoc-containing lichens were maximum in MDF (44\%) followed by WTEF and DDF (28\% each).

Lichens with Trebouxia as photobiont dominate tropical regions with an alternating wet and dry period and include macrolichens in Parmeliaceae and macrolichens and crusts in Bacidiaceae (syn. Ramalinaceae), Physciaceae, and Pertusariaceae [20]. The Trebouxia containing macrolichens in DDF are also equally present in WTEF and MDF, prevailing in our study sites. It includes lichen families such as Parmeliaceae, Physciaceae, and Caliciaceae. Wolseley and Hawksworth [20] reported that the Trebouxia dominated families may occur with increasing dryness and/or disturbance as caused by fire in Thailand.

The Nostoc-containing lichens were found dominant in MDF, while there was equal distribution of Trebouxia containing lichens in all the forest types studied. Lichen taxa with cyanobacteria are more common in the moister forests; species of Collema and Parmeliella were found in DDF as well as SEF, and Leptogium cyanescens was found in all forest types [21]. The lichen species such as Collema actinoptychum, Collema flaccidum, and Collema furfuraceum are confined only to DDF, while Collema subflaccidum is found both in DDF and WTEF. The lichens Leptogium cyanescens and Leptogium denticulatum are found in all forest types as in the case of lichens of northern Thailand forests [21].

Lichen communities of the seasonal evergreen forest (SEF) and tropical mixed deciduous forest (TMDF) are moisture-dependent and shade-adapted, whereas lichen communities of the DDF are adapted to xerophytic conditions and photophilous [21]. This pattern of distribution can be attributed to the sensitivity of the lichen photobiont to drought or high temperatures, which determines the 
TABLE 1: List of lichen species found within the Bolampatti II forest range (Siruvani Hills), Western Ghats, Tamil Nadu, India.

\begin{tabular}{|c|c|c|c|c|c|c|c|c|}
\hline \multirow{2}{*}{$\begin{array}{l}\text { S. } \\
\text { no. }\end{array}$} & \multirow{2}{*}{ Species name } & \multirow{2}{*}{ Family } & \multirow{2}{*}{ Order } & \multirow{2}{*}{$\begin{array}{l}\text { Growth } \\
\text { forms }\end{array}$} & \multirow{2}{*}{ Substratum } & \multicolumn{3}{|c|}{ Forest types } \\
\hline & & & & & & DDF & MDF & WTEF \\
\hline 1 & Bulbothrix isidiza (Nyl.) Hale & Parmeliaceae & Lecanorales & G & 1 & + & - & - \\
\hline 2 & Bulbothrix tabacina (Mont. \& Bosch) Hale & Parmeliaceae & Lecanorales & G & 1 & - & + & - \\
\hline 3 & Canoparmelia aptata (Krempelh.) Elix et Hale & Parmeliaceae & Lecanorales & G & 1 & - & + & - \\
\hline 4 & Cladonia sp. & Cladoniaceae & Lecanorales & $\mathrm{F}$ & 3 & - & + & - \\
\hline 5 & Coccocarpia palmicola (Spreng.) Arv. \& D.J. Galloway & Coccocarpiaceae & Peltigerales & A & 1 & - & + & - \\
\hline 6 & Collema actinoptychum $\mathrm{Nyl}$. & Collemataceae & Peltigerales & $\mathrm{B}$ & 1 & + & - & - \\
\hline 7 & Collema flaccidum (Ach.) Ach. & Collemataceae & Peltigerales & $\mathrm{B}$ & 1 & + & - & - \\
\hline 8 & Collema furfuraceum (Arnold) Du Rietz & Collemataceae & Peltigerales & $\mathrm{B}$ & 1 & + & + & + \\
\hline 9 & Collema rugosum Kremp. & Collemataceae & Peltigerales & $\mathrm{B}$ & 1 & - & + & - \\
\hline 10 & Collema subflaccidum Degel. & Collemataceae & Peltigerales & $\mathrm{B}$ & 1 & + & - & + \\
\hline 11 & Dirinaria applanata (Fée) D.D. Awasthi & Caliciaceae & Teloschistales & $\mathrm{C}$ & 1 & + & - & + \\
\hline 12 & Dirinaria confluens (Fr.) D.D. Awasthi & Caliciaceae & Teloschistales & $\mathrm{C}$ & 2 & - & + & - \\
\hline 13 & Dirinaria consimilis (Stirt.) D.D. Awasthi & Caliciaceae & Teloschistales & $\mathrm{C}$ & 1 & + & - & - \\
\hline 14 & Dirinaria picta (Sw.) Schaer. ex Clem. & Caliciaceae & Teloschistales & $\mathrm{C}$ & 1 & + & + & + \\
\hline 15 & Everniastrum cirrhatum (Fr.) Hale ex Sipman & Parmeliaceae & Lecanorales & $\mathrm{E}$ & 1 & - & + & - \\
\hline 16 & Heterodermia angustiloba (Müll. Arg.) D.D. Awasthi & Physciaceae & Teloschistales & $\mathrm{D}$ & 1 & + & + & - \\
\hline 17 & Heterodermia comosa (Eschw.) Follmann \& Redón & Physciaceae & Teloschistales & $\mathrm{D}$ & 1 & - & - & + \\
\hline 18 & Heterodermia diademata (Taylor) D.D. Awasthi & Physciaceae & Teloschistales & $\mathrm{D}$ & 1 & + & + & + \\
\hline 19 & Heterodermia dissecta (Kurok.) D.D. Awasthi & Physciaceae & Teloschistales & $\mathrm{D}$ & 1 & + & + & + \\
\hline 20 & Heterodermia hypocaesia (Yasuda) D.D. Awasthi & Physciaceae & Teloschistales & $\mathrm{D}$ & 1 & - & + & + \\
\hline 21 & Heterodermia isidiophora (Vain.) D.D. Awasthi & Physciaceae & Teloschistales & $\mathrm{D}$ & 1 & - & + & + \\
\hline 22 & Heterodermia japonica (K. Satô) Swinscow \& Krog & Physciaceae & Teloschistales & $\mathrm{D}$ & 1 & - & + & - \\
\hline 23 & Heterodermia leucomela (Fée) Swinscow \& Krog & Physciaceae & Teloschistales & $\mathrm{D}$ & 1 & - & - & + \\
\hline 24 & Heterodermia microphylla (Kurok.) Skorepa & Physciaceae & Teloschistales & $\mathrm{D}$ & 1 & - & + & + \\
\hline 25 & Heterodermia obscurata (Nyl.) Trevis. & Physciaceae & Teloschistales & $\mathrm{D}$ & 1 & - & + & + \\
\hline 26 & Heterodermia pellucida (D.D. Awasthi) D.D. Awasthi & Physciaceae & Teloschistales & $\mathrm{D}$ & 1 & + & + & + \\
\hline 27 & Heterodermia pseudospeciosa (Kurok.) W.L. Culb. & Physciaceae & Teloschistales & $\mathrm{D}$ & 1 & + & + & + \\
\hline 28 & Heterodermia speciosa (Wulfen) Trevis. & Physciaceae & Teloschistales & $\mathrm{D}$ & 2 & + & + & + \\
\hline 29 & Hypotrachyna coorgiana Patw. \& Prabhu & Parmeliaceae & Lecanorales & A & 1 & - & - & + \\
\hline 30 & Leptogium austroamericanum (Malme) C.W. Dodge & Collemataceae & Peltigerales & $\mathrm{B}$ & 1 & + & + & - \\
\hline 31 & Leptogium azureum (Sw. ex Ach.) Mont. & Collemataceae & Peltigerales & $\mathrm{B}$ & 1 & - & + & - \\
\hline 32 & Leptogium cyanescens (Pers.) Körb. & Collemataceae & Peltigerales & $\mathrm{B}$ & 1 & + & + & + \\
\hline 33 & Leptogium denticulatum F. Wilson & Collemataceae & Peltigerales & $\mathrm{B}$ & 1 & + & + & + \\
\hline 34 & Leptogium isidiosellum (Riddle) Sierk & Collemataceae & Peltigerales & $\mathrm{B}$ & 1 & + & + & - \\
\hline 35 & Leptogium marginellum (Sw.) Gray & Collemataceae & Peltigerales & $\mathrm{B}$ & 1 & - & - & + \\
\hline 36 & Leptogium millegranum Sierk & Collemataceae & Peltigerales & $\mathrm{B}$ & 1 & - & - & + \\
\hline 37 & Leptogium moluccanum (Pers.) Vain. & Collemataceae & Peltigerales & $\mathrm{B}$ & 1 & - & + & + \\
\hline 38 & Leptogium phyllocarpum (Pers.) Mont. & Collemataceae & Peltigerales & $\mathrm{B}$ & 1 & - & + & - \\
\hline 39 & Leptogium pichneum (Ach.) Nyl. & Collemataceae & Peltigerales & $\mathrm{B}$ & 1 & + & - & - \\
\hline 40 & Leptogium ulvaceum (Pers.) Vain. & Collemataceae & Peltigerales & $\mathrm{B}$ & 1 & - & - & + \\
\hline 41 & Lobaria japonica (Zahlbr.) Asahina & Lobariaceae & Peltigerales & A & 1 & - & + & - \\
\hline 42 & Myelochroa aurulenta (Tuck.) Elix \& Hale & Parmeliaceae & Lecanorales & A & 1 & + & + & - \\
\hline 43 & Parmelia direagens Hale & Parmeliaceae & Lecanorales & G & 1 & + & - & - \\
\hline 44 & Parmelina muelleri (Vain.) Hale & Parmeliaceae & Lecanorales & G & 1 & - & - & + \\
\hline 45 & Parmelinella simplicior (Hale) Elix \& Hale & Parmeliaceae & Lecanorales & G & 1 & - & + & + \\
\hline 46 & Parmelinopsis expallida (Kurok.) Elix \& Hale & Parmeliaceae & Lecanorales & G & 1 & + & - & - \\
\hline 47 & Parmelinopsis microlobulata (Awas.) Elix and Hale & Parmeliaceae & Lecanorales & G & 1 & - & - & + \\
\hline 48 & Parmotrema andinum (Müll. Arg.) Hale & Parmeliaceae & Lecanorales & G & 1 & + & - & - \\
\hline 49 & Parmotrema austrosinense (Zahlbr.) Hale & Parmeliaceae & Lecanorales & G & 1 & - & + & - \\
\hline
\end{tabular}


TABle 1: Continued.

\begin{tabular}{|c|c|c|c|c|c|c|c|c|}
\hline \multirow{2}{*}{$\begin{array}{l}\text { S. } \\
\text { no. }\end{array}$} & \multirow{2}{*}{ Species name } & \multirow{2}{*}{ Family } & \multirow{2}{*}{ Order } & \multirow{2}{*}{$\begin{array}{l}\text { Growth } \\
\text { forms }\end{array}$} & \multirow{2}{*}{ Substratum } & \multicolumn{3}{|c|}{ Forest types } \\
\hline & & & & & & DDF & MDF & WTEF \\
\hline 50 & Parmotrema crinitum (Ach.) M. Choisy & Parmeliaceae & Lecanorales & G & 1 & + & - & - \\
\hline 51 & Parmotrema dilatatum (Vain.) Hale & Parmeliaceae & Lecanorales & G & 1 & - & + & - \\
\hline 52 & Parmotrema grayanum (Hue) Hale & Parmeliaceae & Lecanorales & G & 2 & - & + & - \\
\hline 53 & Parmotrema indicum Hale & Parmeliaceae & Lecanorales & G & 1 & - & - & + \\
\hline 54 & Parmotrema melanothrix (Mont.) Hale & Parmeliaceae & Lecanorales & G & 1 & + & - & + \\
\hline 55 & Parmotrema mesotropum (Müll. Arg.) Hale & Parmeliaceae & Lecanorales & G & 1 & + & - & - \\
\hline 56 & Parmotrema permutatum (Stirt.) Hale & Parmeliaceae & Lecanorales & G & 1 & - & + & - \\
\hline 57 & Parmotrema praesorediosum (Nyl.) Hale & Parmeliaceae & Lecanorales & G & $1 \& 2$ & + & - & - \\
\hline 58 & Parmotrema saccatilobum (Taylor) Hale & Parmeliaceae & Lecanorales & G & 1 & + & - & + \\
\hline 59 & Parmotrema tinctorum (Nyl.) Hale & Parmeliaceae & Lecanorales & G & 1 & + & - & - \\
\hline 60 & Parmotrema xanthinum (Müll. Arg.) Hale & Parmeliaceae & Lecanorales & G & 1 & - & - & + \\
\hline 61 & Phaeophyscia hispidula (Ach.) Moberg & Physciaceae & Teloschistales & $\mathrm{C}$ & 1 & + & - & - \\
\hline 62 & Phyllopsora corallina (Eschw.) Müll. Arg. & Ramalinaceae & Lecanorales & A & 1 & + & + & + \\
\hline 63 & Phyllopsora parvifolia (Pers.) Müll. Arg. & Ramalinaceae & Lecanorales & A & 1 & + & + & + \\
\hline 64 & Physcia aipolia (Ehrh. ex Humb.) Fürnr. & Physciaceae & Teloschistales & $\mathrm{C}$ & 1 & + & - & + \\
\hline 65 & Physcia caesia (Hoffm.) Fürnr. & Physciaceae & Teloschistales & $\mathrm{C}$ & 1 & - & - & + \\
\hline 66 & Physcia dilatata $\mathrm{Nyl}$. & Physciaceae & Teloschistales & $\mathrm{C}$ & 2 & + & - & - \\
\hline 67 & Physcia dimidiata (Arnold) Nyl. & Physciaceae & Teloschistales & $\mathrm{C}$ & 1 & + & + & - \\
\hline 68 & Physcia tribacia (Ach.) Nyl. & Physciaceae & Teloschistales & $\mathrm{C}$ & 1 & + & + & + \\
\hline 69 & Physcia tribacioides Nyl. & Physciaceae & Teloschistales & $\mathrm{C}$ & 1 & + & - & - \\
\hline 70 & Pseudocyphellaria aurata (Ach.) Vain. & Lobariaceae & Peltigerales & A & 1 & - & + & - \\
\hline 71 & Pyxine cocoës var. cocoës (Sw.) Nyl. & Caliciaceae & Teloschistales & $\mathrm{C}$ & 1 & + & + & - \\
\hline 72 & Pyxine cocoës var. prominula (Stirt.) D.D. Awasthi & Caliciaceae & Teloschistales & $\mathrm{C}$ & 1 & + & - & - \\
\hline 73 & Pyxine cognata Stirt. & Caliciaceae & Teloschistales & $\mathrm{C}$ & 1 & + & - & - \\
\hline 74 & Pyxine consocians Vain. & Caliciaceae & Teloschistales & $\mathrm{C}$ & 2 & + & - & - \\
\hline 75 & Pyxine himalayensis D.D. Awasthi & Caliciaceae & Teloschistales & $\mathrm{C}$ & 1 & + & - & - \\
\hline 76 & Pyxine meissneriana Nyl. & Caliciaceae & Teloschistales & $\mathrm{C}$ & 1 & + & - & - \\
\hline 77 & Pyxine nilgiriensis D.D. Awasthi & Caliciaceae & Teloschistales & $\mathrm{C}$ & 2 & - & + & - \\
\hline 78 & Pyxine petricola Nyl. & Caliciaceae & Teloschistales & $\mathrm{C}$ & 1 & + & + & - \\
\hline 79 & Pyxine sorediata (Ach.) Mont. & Caliciaceae & Teloschistales & $\mathrm{C}$ & 1 & + & + & + \\
\hline 80 & Pyxine subcinerea Stirt. & Caliciaceae & Teloschistales & $\mathrm{C}$ & 1 & + & - & - \\
\hline 81 & Ramalina baltica Lettau & Ramalinaceae & Lecanorales & I & 1 & - & - & + \\
\hline 82 & Ramalina pacifica Asahina & Ramalinaceae & Lecanorales & I & 1 & + & + & + \\
\hline 83 & Ramalina roesleri (Hochst. ex Schaer.) Hue & Ramalinaceae & Lecanorales & I & 1 & - & + & + \\
\hline 84 & Ramalina sp.2 & Ramalinaceae & Lecanorales & I & 1 & - & + & - \\
\hline 85 & Rimelia reticulata (Taylor) Hale and Fletcher & Parmeliaceae & Lecanorales & G & 1 & + & - & + \\
\hline 86 & Sticta filicina Ach. & Lobariaceae & Peltigerales & G & 1 & - & + & - \\
\hline 87 & Sticta weigelii Isert & Lobariaceae & Peltigerales & G & 1 & - & + & + \\
\hline 88 & Teloschistes flavicans (Sw.) Norman & Teloschistaceae & Teloschistales & I & 1 & - & - & + \\
\hline 89 & Usnea albopunctata Nyl. apud Crombie & Parmeliaceae & Lecanorales & $\mathrm{H}$ & 1 & - & + & - \\
\hline 90 & Usnea bornmuelleri J. Steiner & Parmeliaceae & Lecanorales & $\mathrm{H}$ & 1 & - & - & + \\
\hline 91 & Usnea corallina Motyka & Parmeliaceae & Lecanorales & $\mathrm{H}$ & 1 & - & + & - \\
\hline 92 & Usnea dendritica Stirt. & Parmeliaceae & Lecanorales & $\mathrm{H}$ & 1 & - & + & + \\
\hline 93 & Usnea galbinifera Asahina & Parmeliaceae & Lecanorales & $\mathrm{H}$ & 1 & - & - & + \\
\hline 94 & Usnea orientalis Motyka & Parmeliaceae & Lecanorales & $\mathrm{H}$ & 1 & - & + & + \\
\hline 95 & Usnea pangiana Stirt. & Parmeliaceae & Lecanorales & $\mathrm{H}$ & 1 & - & - & + \\
\hline 96 & Usnea pectinata Stirt. & Parmeliaceae & Lecanorales & $\mathrm{H}$ & 1 & - & + & - \\
\hline 97 & Usnea picta (J. Steiner) Motyka & Parmeliaceae & Lecanorales & $\mathrm{H}$ & 1 & - & - & + \\
\hline 98 & Usnea pictoides G. Awasthi & Parmeliaceae & Lecanorales & $\mathrm{H}$ & 1 & - & + & - \\
\hline
\end{tabular}


TABLE 1: Continued.

\begin{tabular}{|c|c|c|c|c|c|c|c|c|}
\hline \multirow{2}{*}{$\begin{array}{l}\text { S. } \\
\text { no. }\end{array}$} & \multirow{2}{*}{ Species name } & \multirow{2}{*}{ Family } & \multirow{2}{*}{ Order } & \multirow{2}{*}{$\begin{array}{l}\text { Growth } \\
\text { forms }\end{array}$} & \multirow{2}{*}{ Substratum } & \multicolumn{3}{|c|}{ Forest types } \\
\hline & & & & & & DDF & MDF & WTEF \\
\hline 99 & Usnea pseudomontis-fuji Asahina & Parmeliaceae & Lecanorales & $\mathrm{H}$ & 1 & - & + & - \\
\hline 100 & Usnea splendens Stirt. & Parmeliaceae & Lecanorales & $\mathrm{H}$ & 1 & - & - & + \\
\hline 101 & Usnea stigmatoides $\mathrm{G}$. Awasthi & Parmeliaceae & Lecanorales & $\mathrm{H}$ & 1 & - & - & + \\
\hline 102 & Usnea undulata Stirt. & Parmeliaceae & Lecanorales & $\mathrm{H}$ & 1 & - & - & + \\
\hline 103 & Usnea vegae Motyka & Parmeliaceae & Lecanorales & $\mathrm{H}$ & 1 & - & - & + \\
\hline
\end{tabular}

Growth form code: A-foliose; B-foliose gelatinous; C-foliose laciniate; D-foliose ribbon like; E-foliose strap shaped; F-foliose two fold; G-foliose typical; Hfruticose cylindrical; I-fruticose strap shaped. Subsratum code: bark-1; rock-2; soil-3. DDF: dry deciduous forest; MDF: moist deciduous forest; WTEF: western tropical evergreen forest.

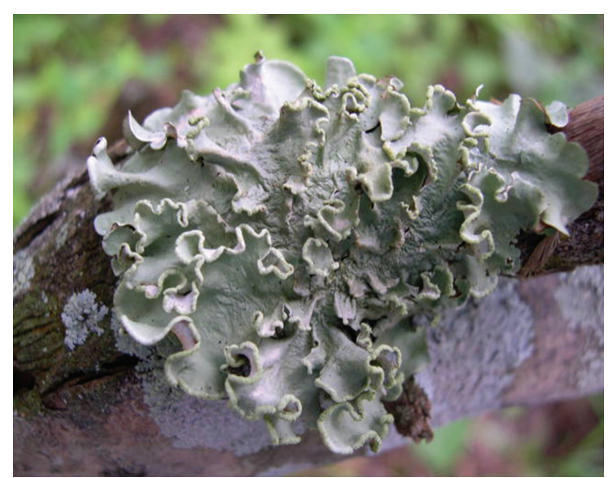

(a)

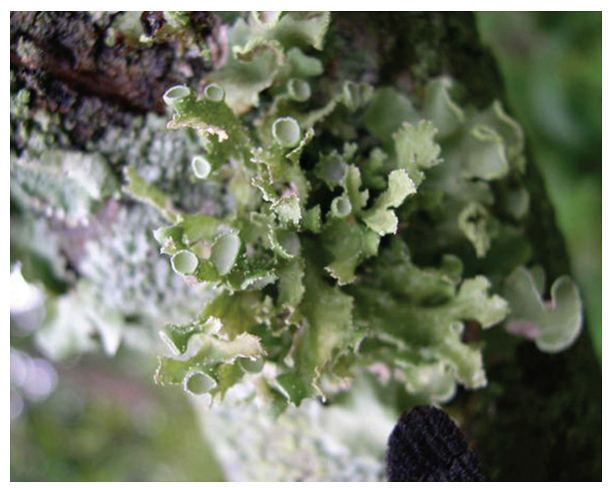

(c)

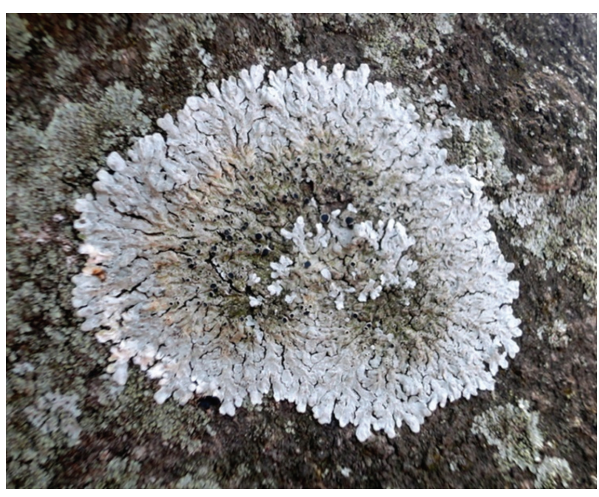

(e)

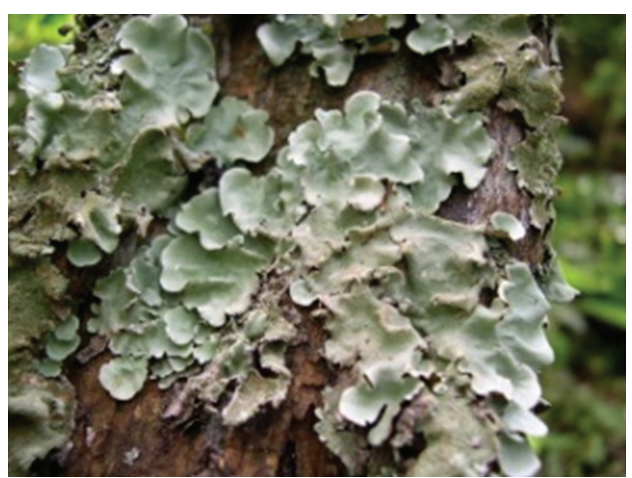

(b)

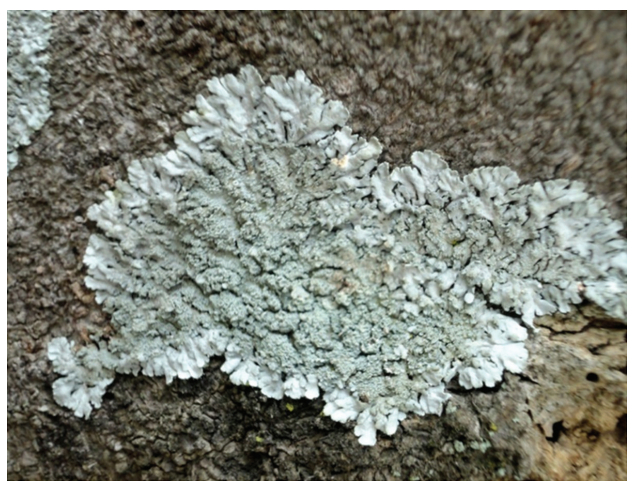

(d)

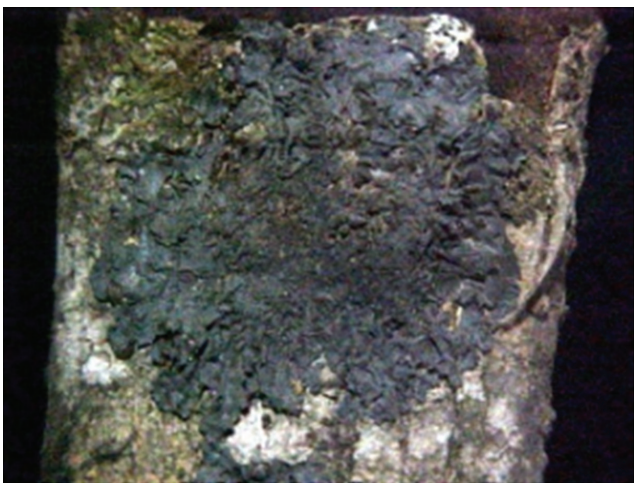

(f)

Figure 2: Examples of lichens examined during this study. (a) Parmotrema praesorediosum, an epiphytic macrolichen, (b) Parmotrema mesotropum (living in an open and dry condition with Trebouxia as photobiont), (c) Ramalina pacifica, a strap shaped lichen, (d) Heterodermia isidiophora, (e) Pyxine cocoes var. cocoes with black ascomata, and (f) Leptogium denticulatum, living in a shade and wet condition with Nostoc as photobiont (a cyanolichen). 
survival of a lichen thallus [21], and the Trebouxia containing lichens are known to survive better in the open and dry $[22,23]$ condition prevailing in the DDF. The seasonal evergreen and tropical mixed deciduous forests of Thailand [24] were reported to contain more numbers of shade-tolerant Trentepohlia containing lichens compared to dry deciduous forests of Thailand. More number of shadetolerant and moisture-dependent Nostoc-containing lichens were observed in MDF type in Bolampatti II forest range also. Lichen families with cyanobacteria and/or chlorococcoid algae include Lobariaceae and Collemataceae, and are more frequent in humid forests. The adaptations to tropical shaded moist conditions are the presence of cyanobacteria and Trentepohlia as photobionts [25].

Bergamini et al. [26] state that macrolichens may be good indicators of lichen species richness because of the highly significant relationships. The species turnover within the study sites was very similar for all lichens and macrolichens. The similar trend was also observed in our study sites. In northern Thailand, the lichen species characteristic of disturbance such as Pyxine consocians and Dirinaria consimilis is also observed in DDF of Bolampatti II forest range. The rate of colonization and growth of these species is an indication of the rate of change [24].

\section{Conclusion}

The present study revealing the occurrence of 103 macrolichens from $197.66 \mathrm{~km}^{2}$ forest area indicates the lichen species richness of the study area and their availability for further ecological monitoring. It is desirable to develop location-specific and lichen-centric conservation strategies using this baseline data to protect the valuable and yet poorly studied ecologically-important group called lichens.

\section{Acknowledgments}

The authors thank Professor M. S. Swaminathan and the Executive Director, MSSRF, for their encouragement and support, Dr. D. K. Upreti for his critical comments on identification and reconfirmation, Principal Chief Conservator of Forests, Tamil Nadu, and District Forest Officer, Coimbatore, for necessary field permit, field guides Mr. P. Chokkalingam, Mr. P. Radhan, Mr. Senthil and Mr. Selvam for their help, Council of Scientific and Industrial Research, New Delhi, and Government of India for the financial support.

\section{References}

[1] J. P. Schmit and G. M. Mueller, "An estimate of the lower limit of global fungal diversity," Biodiversity and Conservation, vol. 16, no. 1, pp. 99-111, 2007.

[2] S. T. Bates, A. Barber, E. Gilbert et al., "A revised catalog of Arizona lichens," Canotia, vol. 6, no. 1, pp. 26-43, 2010.

[3] S. Nayaka, D. K. Upreti, M. Gadgil et al., "Distribution pattern and heavy metal accumulation in lichens of Bangalore city with special reference to Lalbagh garden," Current Science, vol. 84, no. 5, pp. 674-680, 2003.
[4] S. R. Gradstein, "The vanishing tropical rain forest as an environment for bryophytes and lichens," in Bryophytes and Lichens in a Changing Environment, J. W. Bates and M. F. Andrew, Eds., pp. 235-258, Clarendon Press, Oxford, UK, 1992.

[5] H. R. Negi, "On the patterns of abundance and diversity of macrolichens of Chopta-Tunganath in the Garhwal Himalaya," Journal of Biosciences, vol. 25, no. 4, pp. 367-378, 2000.

[6] K. P. Singh and G. P. Sinha, Lichen Flora of Nagaland, Bishen Singh Mahendra Pal Singh, Dehra Dun, India, 1994.

[7] K. P. Singh and G. P. Sinha, "Lichen diversity of the Eastern Himalaya and its conservation," in Himalayan Microbial Diversity, Part 2, (Recent Researches in Ecology, Environment and Pollution), S. C. Sati, J. Saxena, and R. C. Dubey, Eds., vol. 11, pp. 349-359, Today and Tomorrow's Printers and Publishers, New Delhi, India, 1997.

[8] M. Kumar and S. Stephen, "Lichens of Western Ghats-an overview," in Biology of Lichens, K. G. Mukerji, B. P. Chamola, D. K. Upreti, and R. K. Upadhyay, Eds., pp. 297-331, 1999.

[9] J. Wilson, "Working plan for the Bolampatti Range of Coimbatore Central Forest Division," Government of Madras, pp.1-155, 1967.

[10] H. G. Champion and S. K. Seth, A Revised Survey of the Forest Types of India, Manager of Publications, Delhi, India, 1968.

[11] D. D. Awasthi, "Contributions to the lchen flora of India and Nepal-I. The genus Physcia (Ach.) Vain," Journal of the Indian Botanical Society, vol. 39, no. 1, pp. 1-21, 1960.

[12] D. D. Awasthi, A Monograph of the Lichen Genus Dirinaria, vol. 2 of Bibliotheca Lichenologica, J. Cramer, Lehre, Germany, 1975.

[13] D. D. Awasthi and K. P. Singh, "The lichen flora in the environs of Gangotri and Gomukh, India. I-the macrolichens," Indian Journal of Forest Research, vol. 1, pp. 138-146, 1978.

[14] D. D. Awasthi, “Pyxine in India," Phytomorphology, vol. 30, pp. 359-379, 1980.

[15] D. D. Awasthi, "Lichen genus Parmelia in India. II. Subgenera Xanthoparmelia (Vain.) Hale and Melanoparmelia (Hue) Essl," Indian Journal of Forestry, vol. 4, pp. 198-204, 1981.

[16] G. Awasthi, "Lichen genus usnea in India," Journal of the Hattori Botanical Laboratory, vol. 61, pp. 333-421, 1986.

[17] D. D. Awasthi, "A key to the macrolichens of India and Nepal," Journal of the Hattori Botanical Laboratory, vol. 65, pp. 207-302, 1988.

[18] S. Huneck and I. Yoshimura, Identification of Lichen Substances, Springer, Tokyo, Japan, 1996.

[19] D. L. Hawksworth, P. M. Kirk, B. C. Sutton, and D. N. Pegler, Dictionary of the Fungi, CAB International, Wallingford, UK, 8th edition, 1995.

[20] P. A. Wolseley and D. L. Hawksworth, "Adaptations of lichens to conditions in tropical forests of South-East Asia and their taxonomic implications," Blumea, vol. 54, no. 1-3, pp. 29-32, 2009.

[21] P. A. Wolseley and B. Aguirre-Hudson, "The ecology and distribution of lichens in tropical deciduous and evergreen forests of Northern Thailand," Journal of Biogeography, vol. 24, no. 3, pp. 327-343, 1997.

[22] P. Balaji and G. N. Harihran, "Lichen diversity and its distribution pattern in Tropical Dry Evergreen Forest of Guindy National Park (GNP), Chennai," The Indian Forester, vol. 130, no. 10, pp. 1155-1168, 2004.

[23] P. Balaji and G. N. Harihran, "Annonated checklist of lichens of Chennai, Tamil Nadu, India," Phytotaxonomy, vol. 5, pp. 1-7, 2005. 
[24] P. A. Wolseley and B. Aguirre-Hudson, "Fire in tropical dry forests: corticolous lichens as indicators of recent ecological changes in Thailand," Journal of Biogeography, vol. 24, no. 3, pp. 345-362, 1997.

[25] H. J. M. Sipman and R. C. Harris, "Lichens," in Tropical Rain Forest Ecosystems, H. Lieth and M. J. A. Werger, Eds., pp. 303-309, Elsevier Science Publishers B.V., Amsterdam, The Netherlands, 1989.

[26] A. Bergamini, C. Scheidegger, S. Stofer et al., "Performance of macrolichens and lichen genera as indicators of lichen species richness and composition," Conservation Biology, vol. 19, no. 4, pp. 1051-1062, 2005. 

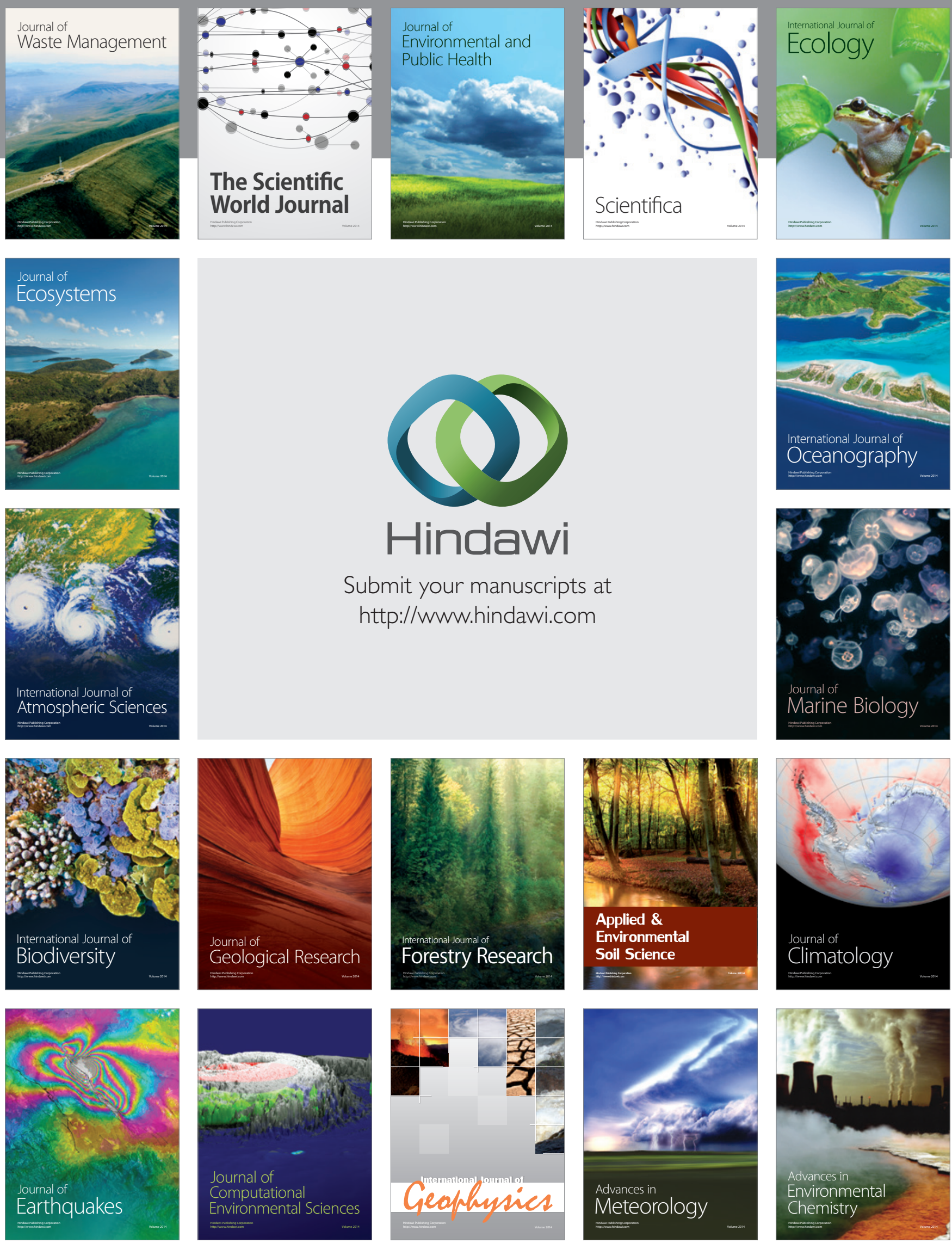